\title{
Is the Primary Visual Cortex a Center Stage for the Visual Phenomenology of Object Size?
}

\author{
Philippe A. Chouinard ${ }^{1}$ and Matthew Ivanowich ${ }^{2}$ \\ ${ }^{1}$ Department of Psychology and the Brain and Mind Institute and ${ }^{2}$ Department of Philosophy and the Brain and Mind Institute, The University of Western \\ Ontario, London, Ontario, N6A 5B8, Canada \\ Review of Pooresmaeili et al.
}

As we watch a car drive away from us, we perceive the car as having the same size even though the image that it projects on the retina becomes smaller. This phenomenon of perceiving an object of a given size as having the same size regardless of viewing distance is known as size constancy. Given the complexity of its computational demands, it is reasonable to assume that high-order areas in the brain would be engaged. Yet, functional magnetic resonance imaging (fMRI) studies in recent years have demonstrated that early sensory structures play a far greater role in size constancy than previously thought. Specifically, fMRI experiments involving optical illusion displays (Murray et al., 2006; Fang et al., 2008) and afterimages (Sperandio et al., 2012) reveal that patterns of activation in the primary visual cortex (V1) more closely reflect the visual experience of an object size than its retinal image size. One view that has been put forth to explain these effects is top-down modulation (Murray et al., 2006; Fang et al., 2008; Sperandio et al., 2012). According to this view, the linear perspective cues in the optical illusion displays providing spatial context in the studies by Murray et al. (2006) and Fang et al. (2008) would have

Received Nov. 21, 2013; revised Dec. 19, 2013; accepted Dec. 20, 2013.

Correspondence should be addressed to Philippe A. Chouinard, Brain and Mind Institute and the Department of Psychology, The University of Western Ontario, 1151 Richmond Street, London, Ontario, N6A 5B8, Canada. E-mail: pchouin@uwo.ca.

DOI:10.1523/JNEUROSCI.4902-13.2014

Copyright $\odot 2014$ the authors $\quad 0270-6474 / 14 / 342013-02 \$ 15.00 / 0$ been processed by other brain areas before feeding into V1. Likewise, the oculomotor cues providing spatial context in the Sperandio et al. (2012) study would have been first processed elsewhere.

In a recent study published in The Journal of Neuroscience, Pooresmaeili et al. (2013) examined the role of $\mathrm{V} 1$ in size perception using fMRI. The authors argued that their paradigm allowed them to examine local responses in V1 in a way that is less prone to top-down modulation than the methods used in the previously mentioned size-constancy experiments. Namely, they posited that the Cornsweet visual stimuli (Fig. 1) they used to drive changes in size perception did not provide any spatial contextual cues, which would have to be first processed by other areas in the brain before feeding into V1.

Pooresmaeili et al. (2013) performed two experiments: (1) a behavioral experiment to determine whether exposure to an adapter stimulus would cause a subsequently presented test stimulus to be perceived as being larger or smaller than an identically sized reference stimulus, and (2) an fMRI experiment to measure BOLD responses in V1 to the same types of stimuli. In the behavioral experiment, a circular test stimulus was shown to participants (Fig. 1). This test stimulus varied in size across trials. A circular adapter of a fixed size was presented at the same spatial location before the test stimulus on some trials but not on others. The participants' task was to indicate whether the test stimulus was smaller or larger than a circular reference presented at the end of every trial. The purpose of this experiment was to construct visual psychometric functions to determine whether or not size perception using Cornsweet stimuli would depend on context, with larger adapting stimuli causing the test stimuli to appear smaller and smaller adapters causing the test stimuli to appear larger. As hypothesized, the test stimuli that were smaller than the adapter were perceived as smaller when preceded by the adapter than when the adapter was not shown (Pooresmaeili et al., 2013, their Fig. 1). Conversely, the test stimuli that were larger than the adapter were perceived as larger when preceded by the adapter than when the adapter was not presented. After demonstrating this proof-of-principle, Pooresmaeili et al. (2013) then performed an fMRI experiment to determine whether V1 activation would correspond to the perceived or retinal image size of the stimuli.

Similar procedures were used for the fMRI experiment, except participants were not asked to perform the comparison task with a reference stimulus (instead, participants passively viewed only the adapters and test stimuli in some conditions and only the test stimuli in other conditions). Consistent with previous fMRI studies that examined V1 activation during size perception in the context of optical illusion displays (Murray et al., 2006; Fang et al., 2008) and afterimages (Sperandio et al., 2012), the authors show that their fMRIadaptation paradigm modulated V1 activity in a manner that mirrored the perceived rather than the retinal image size. This conclusion was based on three key findings. First, the authors found both a reduction in the surface 


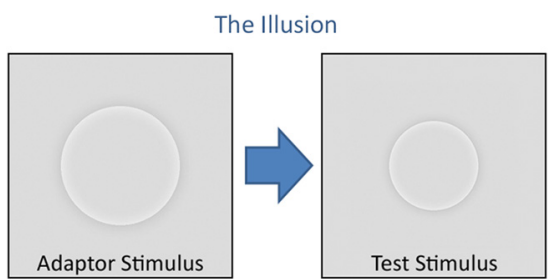

The test stimulus is perceived as smaller than without the adaptor.



The test stimulus is perceived as larger than without the adaptor.

Figure 1. In an adaptation study, the authors presented Cornsweet circles similar to the ones shown here. When presenting an adapter followed by a smaller test stimulus, participants perceived the test stimulus as being smaller, and vice versa.

area of activated V1 cortical tissue when the adapter caused the test stimulus to appear smaller and an augmentation of activated V1 cortical tissue when the adapter caused the test stimulus to appear larger (Pooresmaeili et al., 2013, their Fig. 2C). Second, the authors found that the amount of cortical tissue in V1 activated by the stimuli was strongly correlated with perceived size. Namely, the reported r-statistic was $0.62(p=0.005)$, meaning that that $38 \%\left(R^{2}=0.38\right)$ of the variation in $\mathrm{V} 1$ activation can be accounted for by size perception. Third, when V1 activation was extracted from different regions-of-interest and plotted as a function of retinal eccentric representation, the authors found that the shape of this curve narrowed and widened when subjects perceived the stimuli as being smaller and larger, respectively, as a result of having first seen the adapter (Pooresmaeili et al., 2013, their Fig. 3).

It seems clear that retinotopic activity in V1 reflects the perceived and not the retinal size of the Cornsweet stimuli. Does this imply that V1 is the epicenter for the visual phenomenology of object size? One might be tempted to think so. However, we doubt that Pooresmaeili et al. (2013)'s results should be construed in that way. Consider the following analogy: A computer does not need to encode information in such a way that a representation of a large object takes up more physical space on a hard drive than a representation of a small object along some kind of linear function. Likewise, it would be ecologically inefficient to require a mental representation of an elephant to take up more cortical tissue than that of a mouse. Furthermore, although V1 is necessary for visual experience-as evident by patients with cortical blindness resulting from damage to this brain area (Leopold, 2012) - this does not imply that V1 is sufficient. It then follows that V1 may be necessary for size perception because it is required for the normal functioning of a distributed network of brain areas and not because it acts as the center stage of a Cartesian Theater that then feeds forward for the viewing pleasure of a neural homunculus to see and experience the visual world captured from our eyes (Dennett, 1991).

To be clear, Pooresmaeili et al. (2013) do not argue explicitly in favor of the notion that $\mathrm{V} 1$ is the locus of visual phenomenology for object size. Instead, they show that retinotopic activity in V1 reflects the perceived size of a stimulus and argue that "it is highly possible that these effects originate in V1 and then propagate in a feedforward manner across the cortex" (p. 16006). However, we have a number of reasons to question the degree to which the effects observed by Pooresmaeili et al. (2013) are mediated by feedforward mechanisms.

First, the authors found similar effects in area V2. V1 and V2 are strongly interconnected (Anderson and Martin, 2009), making any distinctions between feedforward and feedback modulation impossible to deduce with $\mathrm{fMRI}$, given its limited temporal resolution. Second, the study does not rule out the possibility that context provided by the adapter could have been mediated by highorder areas as opposed to the authors' proposed gain control mechanisms in V1. The authors did not present $\mathrm{fMRI}$ results beyond extra-striate visual areas and fMRI does not have the temporal resolution to dissociate neuronal activation between the adapter and test stimuli. In fact, in a landmark study, Bar et al. (2006) used magnetoencephalography, which offers far better temporal resolution than fMRI, to demonstrate that context provided by visual information can be processed by frontal lobe structures before feeding back into early visual areas. More importantly, this engagement of frontal lobe structures occurs before the onset of visual perception, which underscores the important role that these areas play in analyzing context for shaping visual phenomenology. With this in mind, the illusory effects reported by Pooresmaeili et al. (2013) could have been driven by a high-order size-contrast effect. Namely, seeing a large adapter might cause one to expect to see a large test stimulus so that when a small test stimulus is presented in its place, it is perceived as smaller than it actually is.

\section{References}

Anderson JC, Martin KA (2009) The synaptic connections between cortical areas V1 and V2 in macaque monkey. J Neurosci 29:1128311293. CrossRef Medline

Bar M, Kassam KS, Ghuman AS, Boshyan J, Schmid AM, Schmidt AM, Dale AM, Hämäläinen MS, Marinkovic K, Schacter DL, Rosen BR, Halgren E (2006) Top-down facilitation of visual recognition. Proc Natl Acad Sci US A 103:449-454. CrossRef Medline

Dennett DC (1991) Consciousness explained. Boston: Brown and Co.

Fang F, Boyaci H, Kersten D, Murray SO (2008) Attention-dependent representation of a size illusion in human V1. Curr Biol 18:17071712. CrossRef Medline

Grill-Spector K, Henson R, Martin A (2006) Repetition and the brain: neural models of stimulus-specific effects. Trends Cogn Sci 10: 14-23. CrossRef Medline

Leopold DA (2012) Primary visual cortex: awareness and blindsight. Annu Rev Neurosci 35:91-109. CrossRef Medline

Murray SO, Boyaci H, Kersten D (2006) The representation of perceived angular size in human primary visual cortex. Nat Neurosci 9:429-434. CrossRef Medline

Pooresmaeili A, Arrighi R, Biagi L, Morrone MC (2013) Blood oxygen level-dependent activation of the primary visual cortex predicts size adaptation illusion. J Neurosci 33:1599916008. CrossRef Medline

Sperandio I, Chouinard PA, Goodale MA (2012) Retinotopic activity in $\mathrm{V} 1$ reflects the perceived and not the retinal size of an afterimage. Nat Neurosci 15:540-542. CrossRef Medline 